\title{
Addendum: Standardizing global gene expression analysis between laboratories and across platforms
}

\author{
Members of the Toxicogenomics Research Consortium \\ Nat. Methods 2, 351-356 (2005).
}

The authors were listed as Members of The Toxicogenomics Research Consortium, with a complete list of authors available in a

Supplementary Note online. A complete list of authors in alphabetical order and their affiliations follows.

Theodore Bammler ${ }^{1}$, Richard P Beyer ${ }^{1}$, Sanchita Bhattacharya ${ }^{2}$, Gary A Boorman ${ }^{3}$, Abee Boyles ${ }^{4}$, Blair U Bradford ${ }^{5}$, Roger E Bumgarner ${ }^{6}$, Pierre R Bushel $^{7}$, Kabir Chaturvedi ${ }^{8}$, Dongseok Choi ${ }^{9}$, Michael L Cunningham ${ }^{3}$, Shibing Deng ${ }^{5}$, Holly K Dressman ${ }^{4}$, Rickie D Fannin $^{7}$, Fredrico M Farin ${ }^{1}$, Jonathan H Freedman ${ }^{4}$, Rebecca C Fry ${ }^{2}$, Angel Harper ${ }^{8}$, Michael C Humble ${ }^{10}$, Patrick Hurban ${ }^{8}$, Terrance J Kavanagh ${ }^{1}$, William K Kaufmann ${ }^{5}$, Kathleen F Kerr ${ }^{11}$, Li Jing ${ }^{12}$, Jodi A Lapidus ${ }^{9}$, Michael R Lasarev ${ }^{13}$, Jianying Li ${ }^{7}$, Yi-Ju Li $^{4}$, Edward K Lobenhofer ${ }^{8}$, Xinfang Lu ${ }^{14}$, Renae L Malek ${ }^{15}$, Sean Milton ${ }^{2}$, Srinivasa R Nagalla ${ }^{14}$, Jean P O’Malley ${ }^{14}$, Valerie S Palmer ${ }^{13}$, Patrick Pattee ${ }^{14}$, Richard S Paules ${ }^{7}$, Charles M Perou ${ }^{5}$, Ken Phillips ${ }^{8}$, Li-Xuan Qin ${ }^{11}$, Yang Qiu ${ }^{8}$, Sean D Quigley ${ }^{1}$, Matthew Rodland ${ }^{14}$, Ivan Rusyn ${ }^{5}$, Leona D Samson ${ }^{2}$, David A Schwartz ${ }^{4}$, Yan Shi ${ }^{5}$, Jung-Lim Shin ${ }^{12}$, Stella O Sieber ${ }^{7}$, Susan Slifer ${ }^{4}$, Marcy C Speer ${ }^{4}$, Peter S Spencer ${ }^{13}$, Dean I Sproles ${ }^{13}$, James A Swenberg ${ }^{5}$, William A Suk ${ }^{10}$, Robert C Sullivan ${ }^{12}$, Ru Tian ${ }^{5}$, Raymond W Tennant ${ }^{7}$, Signe A Todd ${ }^{14}$, Charles J Tucker ${ }^{7}$, Bennett Van Houten ${ }^{10}$, Brenda K Weis ${ }^{10}$, Shirley Xuan ${ }^{2}$ \& Helmut Zarbl ${ }^{12}$

${ }^{1}$ Department of Environmental and Occupational Health Sciences and the Center for Ecogenetics and Environmental Health, University of Washington, Box 357234, Seattle, Washington 98195-7234, USA. ${ }^{2}$ Biological Engineering Division, Center for Environmental Health Sciences, Massachusetts Institute of Technology, 77 Massachusetts Avenue, 56-235 Cambridge, Massachusetts 02139, USA. ${ }^{3}$ National Toxicology Program, National Institute of Environmental Health Sciences, 111 TW Alexander Drive, Research Triangle Park, North Carolina 27709, USA. ${ }^{4}$ Duke University Medical Center, Department of Medicine, DUMC 2629, Room 275 MSRB, Research Drive, Durham, North Carolina 27710, USA. ${ }^{5}$ Lineberger Comprehensive Cancer Center, University of North Carolina, CB\#7295, Chapel Hill, North Carolina 27599, USA. ${ }^{6}$ Department of Microbiology, Box 358070, University of Washington, Seattle, Washington 98195, USA. ${ }^{7}$ National Center for Toxicogenomics, National Institute of Environmental Health Sciences, 111 TW Alexander Drive, Research Triangle Park, North Carolina 27709, USA. ${ }^{8}$ Icoria, Inc., 108 TW Alexander Drive, Building 1A, Research Triangle Park, North Carolina 27709, USA. ${ }^{9}$ Division of Biostatistics, School of Public Health and Preventive Medicine, School of Medicine, Oregon Health \& Science University, Portland, Oregon 97239, USA. ${ }^{10}$ Division of Extramural Research and Training, National Institute for Environmental Health Sciences, 111 TW Alexander Drive, Research Triangle Park, North Carolina 27709, USA. ${ }^{11}$ Department of Biostatistics, University of Washington, Box 357234, Seattle, Washington 98195-7234 USA. ${ }^{12}$ Fred Hutchinson Cancer Research Center, 1100 Fairview Ave North, Mailstop C1-1015, Seattle, Washington 98109, USA. ${ }^{13}$ Center for Research on Occupational and Environmental Toxicology, Oregon Health \& Science University, Portland, Oregon 97239, USA. ${ }^{14}$ Center for Biomarker Discovery, Department of Pediatrics, Oregon Health \& Science University, Portland, Oregon 97239, USA. ${ }^{15}$ The Institute for Genomic Research, Rockville, Maryland, USA.

\section{Corrigendum: Multiple-laboratory comparison of microarray platforms}

Rafael A Irizarry, Daniel Warren, Forrest Spencer, Irene F Kim, Shyam Biswal, Bryan C Frank, Edward Gabrielson, Joe G N Garcia, Joel Geoghegan, Gregory Germino, Constance Griffin, Sara C Hilmer, Eric Hoffman, Anne E Jedlicka, Ernest Kawasaki, Francisco MartínezMurillo, Laura Morsberger, Hannah Lee, David Petersen, John Quackenbush, Alan Scott, Michael Wilson, Yanqin Yang, Shui Qing Ye \& Wayne Yu

Nat. Methods 2, 345-349 (2005).

The GEO accession number for the array data is GSE2521. 